\title{
BMJ Open Remuneration of primary dental care in England: a qualitative framework analysis of perspectives of a new service delivery model incorporating incentives for improved access, quality and health outcomes
}

Peter G Robinson, ${ }^{1}$ Gail V A Douglas, ${ }^{2}$ Barry J Gibson, ${ }^{3}$ Jenny Godson, ${ }^{2,4}$ Karen Vinall-Collier, ${ }^{2}$ Sue Pavitt, ${ }^{2}$ Claire Hulme ${ }^{5}$

To cite: Robinson PG, Douglas GVA, Gibson BJ, et al. Remuneration of primary dental care in England: a qualitative framework analysis of perspectives of a new service delivery model incorporating incentives for improved access, quality and health outcomes. BMJ Open 2019;9:e031886. doi:10.1136/ bmjopen-2019-031886

- Prepublication history for this paper is available online. To view these files, please visit the journal online (http://dx.doi. org/10.1136/bmjopen-2019031886).

Received 29 May 2019 Revised 16 August 2019 Accepted 21 August 2019
Check for updates

(c) Author(s) (or their employer(s)) 2019. Re-use permitted under CC BY-NC. No commercial re-use. See rights and permissions. Published by BMJ.

For numbered affiliations see end of article.

Correspondence to

Dr Claire Hulme;

C.T.Hulme@exeter.ac.uk

\section{ABSTRACT}

Objective This study aimed to describe stakeholder perspectives of a new service delivery model in primary care dentistry incorporating incentives for access, quality and health outcomes.

Design Data were collected through observations, interviews and focus groups.

Setting This was conducted under six UK primary dental care practices, three working under the incentive-driven contract and three working under the traditional activitybased contract.

Participants Observations were made of 30 dental appointments. Eighteen lay people, 15 dental team staff and a member of a commissioning team took part in the interviews and focus groups.

Results Using a qualitative framework analysis informed by Andersen's model of access, we found oral health assessments influenced patients' perceptions of need, which led to changes in preventive behaviour. Dentists responded to the contract, with greater emphasis on prevention, use of the disease risk ratings in treatment planning, adherence to the pathways and the utilisation of skill-mix. Participants identified increases in the capacity of practices to deliver more care as a result. These changes were seen to improve evaluated and perceived health and patient satisfaction. These outcomes fed back to shape people's predispositions to visit the dentist. Conclusion The incentive-driven contract was perceived to increase access to dental care, determine dentists' and patients' perceptions of need, their behaviours, health outcomes and patient satisfaction. Dentists face challenges in refocusing care, perceptions of preventive dentistry, deployment of skill mix and use of the risk assessments and care pathways. Dentists may need support in these areas and to recognise the differences between caring for individual patients and the patient-base of a practice.

\section{INTRODUCTION}

Commissioning of National Health Service (NHS) dentistry in England is moving away from volume-based contracts with payment
Strengths and limitations of this study

- While participant numbers are modest, staff were purposively sampled across a range of skill mix so similar numbers were observed.

- Patients and 'non-patients' were recruited - the latter to include people who may not engage with local dental care services.

- There will inevitably be a degree of bias given that all the practices were self-selected.

- The model of access was broadly sustained in the data but might be enhanced by greater conceptual clarity.

- Although the new practices increased access, further work is required to understand how best to promote and encourage appropriate dental service attendance.

for units of dental activity (UDA) to an approach that rewards quality and oral health improvement alongside activity. ${ }^{1}$ Payments recognise prevention and reward the contribution of the dental team to improved oral health, reflected in patient progression along care pathways, adherence to nationally agreed clinical guidelines and the achievement of expected outcomes. ${ }^{1}$ The Department of Health and Social Care (DHSC) dental contract reform programme opened a series of pilots in 2011, subsequently followed by ongoing prototype systems, to explore a shift from treatment and repair to prevention and improved oral health via a new clinical pathway and new remuneration models. ${ }^{2}{ }^{3}$ While the impact of these contracts on process has been investigated, limited evidence exists on their effect on oral 
health outcomes and patient, commissioner and workforce acceptability.

The care pathway in the pilots and prototypes begins with an oral health assessment (OHA) ${ }^{3}{ }^{4}$ designed to enable more prevention within personalised care plans, taking into account patients' social and dental histories and clinical status. Patients are then advised of their oral risk status using a red/amber/green (RAG) rating, given preventive advice and a follow-up or review based on their risk status. The RAG tool allows dentists to perform detailed and consistent assessments that, in turn, direct delivery of care appropriate to needs and aids communication with patients. Evaluation of the pilots reported patient and practitioners' views of the new clinical pathway to be strongly supportive. ${ }^{5}$ However, evidence on the effectiveness of use of contracting and incentives in health providers is still emerging and is mixed. ${ }^{6-9}$ Within dentistry, changes to incentive structures towards a fee for service model increased activity and influenced dentists to target UDAs by shifting towards treatments with high rewards rather than prescribing on the basis of need. ${ }^{1011}$ However, a recent review found low-level evidence and concluded that changes to remuneration may change clinical activity in primary care dentistry but further experimental research is needed. ${ }^{12}$ Furthermore, there is little literature regarding care pathways in primary dental care. ${ }^{13}$ Findings from the national dental contract pilots suggested small improvements in risk reduction over the short term. ${ }^{14}$ The new prototypes are undergoing evaluation. ${ }^{14}$

Another potential advantage of a new dental contract would be more effective use of skill mix. Dental hygienists/therapists may provide treatments such as scaling and polishing, oral health promotion, fissure sealants and fillings on all patients and pulp treatment/stainless steel crowns and extractions on children. The potential contribution of dental therapists to reduce costs and increase access to care is considerable. While there are few hard data to support skill mix in dentistry, ${ }^{15}$ approximately $60 \%-70 \%$ of dentists are prepared to consider employing a therapist, although some dentists remain unclear of their roles. ${ }^{16-18}$

In 2007, a new incentive-driven contract (INCENTIVE) intended to promote evidence-based preventive interventions, widen access to dentistry and encourage the use of skill mix (skill mix in dentistry describes a model which might include, for example, dentists, dental therapists, dental hygienists and dental nurses) was introduced in three primary care dental practices in West Yorkshire. The practices were in areas of high oral health need and with high demand for NHS dental care. While it predated the national dental contract pilots and prototypes, the specification of the INCENTIVE model reflected the same ethos and recommendations, with several features identical. Like the prototypes, the INCENTIVE model blended incentives to demonstrate quality, oral health improvements and volume of service. Most of its contract value $(60 \%)$ was attributed to the delivery of UDAs, with the remainder equally divided between quality (including systems, processes and infrastructure) and oral health improvement via implementation of Delivering Better Oral Health. ${ }^{19}$ The model also employed the same OHA care pathway.

Our overall aim was to evaluate the incentive-driven model of dental service provision implemented in West Yorkshire in the North of England. Our objectives were to (1) explore stakeholder perspectives of the new service delivery model, (2) assess its effectiveness and (3) assess its cost-effectiveness. This manuscript reports on the first objective; details of the effectiveness and cost-effectiveness evaluation can be found elsewhere. ${ }^{20} 21$

\section{METHODS}

This qualitative study focused on the three INCENTIVE practices and three comparator dental practices working under traditional UDA contracts (TRADITIONAL practices). The TRADITIONAL were matched with the INCENTIVE practices by deprivation index, age profile, size of practice and ethnicity.

Data were collected in focus groups and semistructured interviews, supplemented with observations of dental appointments of the delivery of dental care. Purposive sampling via a sampling matrix supported recruitment of participants with different experiences of the model. The three stakeholders groups were lay people (patients and non-patients), dental teams (dental practitioners, dental care professionals and practice managers) and a service commissioner. The sampling matrix for the public and patient group included criteria linked to demographic factors (age, gender, ethnicity, socioeconomic status), risk category and treatment need.

Encounters were observed in two INCENTIVE and two TRADITIONAL practices. Staff were sampled so that similar numbers (15 each) of dentists and dental hygienist/therapists were observed. All eligible adult patients (18 years or older) with appointments on the observation day were invited to participate. Two weeks before their appointments, patients were sent a letter informing them of the study, a study information leaflet and consent form. Patients who expressed interest in participating were given the opportunity to ask any questions and give consent on the day of their appointment. The 'non-participant' observer (a researcher whose background lies in sociology) attended all appointments passively at a distance close enough to hear the conversation to take comprehensive field notes. A brief analysis of observations was conducted as soon as possible after the observation (the same or following day).

Observations were followed by interviews on the same day with four dentists and four dental hygienists/therapists. Staff commented on the encounters and shared their views on what had taken place. Questions asked at the post-observation interview were influenced by the nature of the activity in the encounters, the team member's attitude, expectations and impressions, and 
reflections of the experience. Interviews were recorded and transcribed verbatim.

Interviews were also conducted with patients, other lay people, commissioners and dental team members. Lay people were recruited in two ways: (1) practices mailed information packs to patients; (2) focus groups were held with groups attending a community centre, including one for parents with young children and another for older residents. In addition, snowball sampling entailed existing participants passing the study information and the researcher's contact details on to acquaintances. Participants should be aged 16 years and older. People with no natural teeth were excluded.

Interviews and focus groups followed a topic guide, partly informed by the theoretical framework and supplemented with themes that emerged from the observations and previous interviews. Interviews with dental team members took place at the dental surgery, while interviews with patients took place in patients' homes. All were recorded and transcribed verbatim. Interviews lasted between 15 and $70 \mathrm{~min}$.

The Andersen behavioural model of access was employed as the theoretical framework for the qualitative analysis (figure 1). The model sees access as 'the use of personal health services and everything that facilitates or impedes their use. ${ }^{22}$

The model has evolved in line with advances in understanding to incorporate the interaction between individual, healthcare system and external environment. ${ }^{22-24}$ Later versions introduced health and patient satisfaction as desirable outcomes, said to be determined by predisposing and enabling factors, behaviours and need. Many studies in healthcare, including dentistry, support its use. $^{25} 26$

People may be predisposed to accessing care as a result of their demographic factors, social environment and beliefs. Enabling factors include policies, facilities, staff finance and the organisation of services that might influence utilisation. ${ }^{24}$ From this perspective, the INCENTIVE contract is an enabling factor with policy, financial and organisational facets. ${ }^{13}{ }^{19}$ Health needs may include health education, disease prevention, diagnosis, treatment, rehabilitation and palliative care. Andersen distinguished between evaluated (professionally defined or normative) and perceived (personally defined or felt) need. In turn, these components could influence

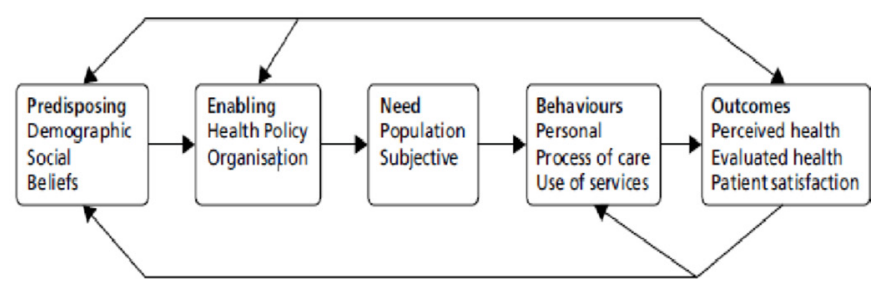

Figure 1 Andersen's behavioural model of access (adapted from Baker ${ }^{26}$ ). personal and professional health behaviours. Personal behaviours are activities that shape health status, such as oral hygiene, diet and tobacco use. Health service use is treated as behaviour in itself. Professional behaviours relate to processes such as health education, communication and prescribing. The maintenance and improvement of health should be the primary outcome of access, thus the outcomes are (patient) perceived and (clinician) evaluated health status and patient satisfaction. An important feature of the model is its recursive nature with feedback loops so that the outcomes of access may influence future predisposing and enabling factors, population needs and use of services.

Framework analysis was used to induce the results from the original accounts within the structured policy focus of the research. ${ }^{27}$ Our intention was to explore the effect of the contract as an enabling factor, interacting with other stages of the model. While Andersen's model guided the analysis, it was refined as required to identify the thematic framework. Data were indexed and charted under subheadings derived from the framework to enable a process of constant comparison across themes and cases. Thus, the framework analysis served to either confirm or challenge the model, with deviant case analysis used to add new categories or revise it. The validity of the findings was supported by discussion of interim and final results for triangulation and corrections with participants in focus groups. The results were also compared against existing knowledge, such as the evaluations of the NHS Dental Contract Pilots. ${ }^{5}$

\section{Patient and public involvement}

Throughout the research cycle, patient contributors worked as integral members of the research team from conception of the research idea to shape our research questions and aid delivery, project management and final data interpretation through to reporting. They ensured our research was of relevance to patients and the NHS; specifically patient contributors: helped identify and prioritise the research questions and develop the research design (eg, the sampling matrix was co-designed); were members of the study advisory group; and co-developed participant information leaflets and dissemination materials.

\section{RESULTS}

Data were collected between August 2012 and February 2014. Observations were made of 30 dental appointments. Eighteen lay people, 15 dental team staff ( 4 traditional practice dentists, 8 INCENTIVE dentists, 2 practice managers and a dental therapist) and a member of the commissioning team took part in the interviews and focus groups. The results are presented in two stages. First, the major themes in the data are outlined. Second, the interactions between enabling and other factors are described.

\section{Major themes}

The Andersen Framework was largely sustained in the data, with the only revision being the addition of trust 
as an outcome of access. Predisposing factors could be characterised as demographic and social characteristics and beliefs. For example, family commitment could facilitate or hamper service utilisation. Enabling factors fell into three subthemes of health policy, finance and organisations. The influence of health policy between the extremes of the changes associated with implementation of the INCENTIVE model right through to an apparent lack of policy in some TRADITIONAL practices. A key part of the contract was dentists' remuneration. Traditional models were problematic for complex cases, whereas INCENTIVE practices focused on the costs of OHAs and building relationships with patients that would enable more prevention. Computing problems featured as organisational factors, and in particular the practice software, which had not been adapted to INCENTIVE.

Evaluated and environmental need and population health indicators were manifest in the data. Two localities in the study are characterised by material deprivation, poor oral health and long-standing undersupply of care. Unsurprisingly, this influenced dental treatment needs.

An example of professional behaviour involved formally assessing patients' risk of disease. Dental team members commented on the relative imprecision of the traffic light (RAG) system and its three categories. Indeed, attitudes and practices towards prevention varied appreciably among the dental teams, with one dentist noting: I do find it hard to talk about their health - I'm trained to drill and fill.

Health outcomes and satisfaction appeared in the data, as did the concept of trust. One INCENTIVE patient noted: I do trust them here - they treated me, gave me root canal treatment and saved my tooth, without them, I'd have been minus a few teeth and my appearance would not have been good.

\section{Interactions}

The INCENTIVE contract changes the finance and organisation of dental practices to implement health policy. Its effects can therefore be seen as interactions between these enabling factors and other stages in the model. This can be seen in enabling and predisposing factors and need. INCENTIVE practices had been located based on "a robust oral health needs assessment prior to commissioning. these practices and we'd looked very closely at equity in terms of access to dental care" (Service Commissioner). For some participants, the INCENTIVE practices marked a shift from no dental care, whereas others moved from private to NHS provision. The new services suited participants' needs in terms of location, personnel and ease of getting an appointment.

Effects of the INCENTIVE contract were evident on the processes of care, personal health practices and the use of personal health resources. In turn, the process of delivering care appeared to be affected in three ways: (1) by the use of the care pathway underpinned by the risk assessment/RAG; (2) by increasing prevention communication and (3) multidisciplinary approach through wider use of skill mix. Of interest the communication of the RAG ratings was not always apparent in the observations and was being used solely by some of the clinicians to document progress.

Participants reflected on their experiences with the pathways. Benefits included the clear link between the risk assessment and care pathways. The INCENTIVE contract embedded DHSC guidance ${ }^{26}$ so that prevention became valued standard procedure. Practitioners felt that it gave them time and space to care for patients. For example, one INCENTIVE dentist noted: Red, amber or green and then they do get the fluoride varnish, the smoking cessation and alcohol use is being taken automatically. This could be contrasted directly with the TRADITIONAL practices. There was an example of where the focus of care in the incentive contract had penetrated a TRADITIONAL practice, causing them to reflect on their processes of care: We are pushed towards UDAs rather than improving oral health... The prevention emphasis is an issue - we are expected to talk about perio disease and smoking and diet and have to squeeze that in.

The INCENTIVE practices were not mandated to use multidisciplinary teams, but did so to deliver preventatively focused care. One approach was for dentists to examine patients and formulate treatment plans, but some practices did not deduct the value of the delegated treatment from dentists' incomes:

They readily work with each other. I mean our therapist and the hygienist are generally busy the whole day, ... we pay them on a fixed rate and the therapists are very happy with that because they've got full time work, they're busy, the associates are happy because they're not having to pay for them ..., the patients get benefit because they get access to a therapist, ... it may cost more for us to do it but it's a more sensible way of running a business because everybody is working together for the same aim.

The new contractual arrangements were seen to influence personal health practices and the use of personal health resources. They also influenced outcomes of perceived and evaluated health and patient satisfaction. Moreover, the interactions could ripple throughout the model to have far-reaching effects. For example, the RAG ratings could influence patients' perceptions of their own needs, leading to personal behaviour changes and satisfaction (an outcome). As one INCENTIVE patient said: $I$ think it's good because if you know, if someone says to you, you know on this rating you are more at risk, you're more likely to do something about it aren't you, as opposed to someone not saying anything to you... For dentists, use of the ratings to determine recall intervals liberated more time for the process of care and allowed observation of increased health but influenced patient satisfaction both positively and negatively, which suggests a need to reconcile contrasting views: the ability to increase access and longer intervals between assessments. In a wholly positive example, one INCENTIVE patient satisfied with her own care encouraged her partner to attend so that professional behaviour 
enhanced satisfaction to change predisposing factors to increase access to care.

\section{DISCUSSION}

This study has described stakeholders' views of the INCENTIVE contracting arrangements. The ratings from OHAs influenced patients' perceptions of need, which led to changes in preventive behaviour. Dentists had responded to the contract in the desired direction with greater emphasis on prevention, use of the ratings in treatment planning, adherence to the pathways and the utilisation of skill mix. Participants identified increases in the capacity of practices to deliver more care as a result. These changes were seen to improve evaluated and perceived health and patient satisfaction. Although participant numbers were relatively modest, these findings are compatible with the first year evaluations of the NHS dental contract pilots where almost three-quarters of patients said they had a better understanding of their oral health and had changed their behaviour. ${ }^{5}$ Furthermore, analysis of OHAs and reviews suggested that RAG ratings improved for small numbers of patients, within 2years. ${ }^{4}$ Better health and satisfaction increased the predisposition to visit the dentist. These findings demonstrate the potential for a new contract to increase access and to improve health.

While these data are encouraging for continued contract reform, several areas were identified as requiring more consideration, where NHS England, dental teams and the public may need more support. The practices had been placed in areas of high need. As well as meeting immediate needs, the perception of low availability of care may also be a barrier to access in areas that have been underserved in the past. Evidence of new services is therefore needed to break this cycle. The results also demonstrate direct benefits of the needs-led local service commissioning of the 2006 dental contract. ${ }^{28}{ }^{29}$ Such local knowledge is less well used in the current NHS England single operating model for practice commissioning, ${ }^{30}$ which may render the system less responsive.

Participants at INCENTIVE practices reported professional and lay preventive behaviours leading to better evaluated and perceived health. However, there were concerns over offering preventive advice, the complexities of accounting for the patient's context, the time this took and the difficulties of effective prevention, especially in areas with high levels of disease. These aspects of dentistry are often presented as problems, sometimes beyond the scope of practitioners, rather than part of their job. ${ }^{16}$ This is a key issue if dentistry is to refocus on prevention. These and other data indicate that change is possible if it is encouraged by the right contractual model. Education may also be required to support contractual drivers. Neither one alone is likely to be sufficient. A Cochrane review $^{31}$ concluded that educational meetings had a small effect on professional practice and health outcomes but the effects were likely to be smaller still for complex behaviours. Both a systematic review of incentives to follow best practice in healthcare and a Cochrane review of the effect of remuneration on primary care dentists' behaviour cited within it $^{12} 32$ concluded that financial incentives can have a 'modest' effect on improving the quality of healthcare.

The emphasis on OHAs and pathways was a key feature of INCENTIVE and the DHSC contract pilots and subsequent prototypes. ${ }^{5}$ Some patients were not aware of the RAG ratings; others perceived them to alert them to their preventive needs and to be a motivator. We specifically enquired about the RAG ratings when triangulating the data in focus groups with dental staff. There was near universal use of the ratings as a decision aid (as evident in the data), but their use in patient communication had decreased over time. Dental teams may need clarification of whether and how the ratings are supposed to be communicated to patients. The small number of rating categories concerned some dentists, especially for patients with immutable risk factors such as general health problems. Dentists' concerns might be alleviated by the addition of new categories. Alternatively, they may become used to this system and gain confidence in overriding the rules of the pathway. Their reluctance to do this may stem from the requirement to justify doing so. Dentists who engaged in the contract pilots requested reassurance about exercising clinical judgement in deviating from recommendations, ${ }^{33}$ first that there would be no medicolegal repercussions provided there was evidence of clinical justification; and second, that they would not be penalised contractually.

Dental therapists increased the availability of care and patient satisfaction. While contract reform is seen to play a key role in use of wider dental team skill mix and enabling different models of care, ${ }^{34}$ use of skill mix is exquisitely sensitive to contracting and practice finance. ${ }^{35}$ Practices had increased their utilisation of wider skill mix by not reducing the payments to dentists who delegated care. Paying two staff members for the shared treatment incentivised referral across the team and also liberated dentists' time for patient OHAs and more remunerative complex treatments. The impact of funding arrangements on dental practice is well known, and dentists must reconcile the business and other elements of their practices. ${ }^{36}$ Dentists could see how the new contract carried the potential for greater value for money and reduce unnecessary treatment, but there were concerns that the time and costs of the OHAs had been underestimated. This was also a concern in the dental contract pilots and was evident as reduced patient access. ${ }^{33} 37$ This financial risk may be particularly relevant to new practices. The pilots were conducted in existing practices and experienced falls in access. However, the challenges may be even more severe in new practices (such as the INCENTIVE practices), where all the patients required initial assessments and were more likely to have high treatment needs necessitating more visits. This consideration goes further than dentists' incomes as concerns about costs 
were seen to influence practitioner behaviour. These concerns support the notion of assigning contract values according to patient needs, which in the contract prototypes is achieved by weighing the capitation element by patient characteristics of age and deprivation status. ${ }^{37}$

The Andersen model provided a useful taxonomy for the data and allowed identification of the effects of the new contract. This fit is unsurprising as the model was developed over a 40-year period and remains among the most widely cited models of access to healthcare. ${ }^{2324} 2638$ Theoretical models may be tested empirically or by looking for logical coherence. ${ }^{39}$ The Andersen model is somewhat general with overlapping dimensions and others (eg, social capital) not explicitly incorporated. ${ }^{26}$ Very little is said about how demographic variables may exert effects and why. ${ }^{38} \mathrm{~A}$ new factor in these data ('Trust') is not explicit in the model but could be regarded as both a belief and an outcome of care. ${ }^{39}$ Dimensions also overlap in the model where dissimilar concepts are grouped together (personal health practices and health service use, perceived and evaluated health and satisfaction). This is important because empirical testing demands careful specification of inclusive relationships and, to a certain extent, this confounded testing of this model. ${ }^{38}$ One consequence of this might be that the model yields very different results when cross national comparisons are made. ${ }^{40}$

\section{CONCLUSION}

The incentive-driven contract influenced access to dental care. Participants associated it with more access, greater use of skill mix and improved health outcomes. These outcomes fed back to shape predispositions to visit the dentist.

The policy context in which the INCENTIVE study was funded has remained remarkably constant ever since. The Steele Report of $2009^{1}$ advocated commissioning to align dentistry with the rest of NHS services and to commission for health outcomes; to develop contracts rewarding activity, quality and oral health improvement. It recommended that payments explicitly recognise prevention and reward the contribution of the dental team to improvements to oral health, reflected in patient progression along the pathway, adherence to clinical guidelines and the achievement of expected outcomes. ${ }^{1}$ The contract prototypes now being tested ${ }^{37}$ retain the same ethos of shifting NHS dentistry towards prevention and oral health rather than treatment and repair through a new clinical pathway and new remuneration models. Despite predating the Steele report, the INCENTIVE contracts were forerunners of these new incentive-driven contracts. Thus, our findings remain directly relevant to the evolution of the NHS dental contract.

\section{Author affiliations}

${ }^{1}$ School of Oral and Dental Sciences, University of Bristol, Bristol, UK

${ }^{2}$ School of Dentistry, University of Leeds, Leeds, UK
${ }^{3}$ School of Clinical Dentistry, University of Sheffield, Sheffield, UK

${ }^{4}$ Dental Public Health, Public Health England, Leeds, UK

${ }^{5}$ Health Economics Group, University of Exeter, Exeter, UK

Acknowledgements The study team would like to thank Mel Hall and Jenny Porritt, members of the study team involved in data collection and analysis. We would also like to thank the study participants. Department of Health disclaimer: this paper presents independent research commissioned by the National Institute of Health Research (NIHR). The views and opinions expressed by authors in this publication are those of the authors and do not necessarily reflect those of the NHS, the NIHR, Medical Research Council, Central Commissioning Facility, NIHR Evaluation, Trials Studies Co-ordinating Centre (NETSCC), the Health Services and Delivery Research programme or the Department of Health.

Contributors PGR led the qualitative study. He designed the study, collected data, oversaw the analysis and interpretation and drafted the paper. GVAD designed the study, collected data, and input to the interpretation and analysis and to the paper. BJG designed the study, collected data, and input to the interpretation and analysis and to the paper. JG designed the study, input to the interpretation and analysis and to the paper. KV-C designed the study, collected data, and input to the interpretation and analysis and to the paper. SP designed the study and input to the interpretation and analysis and to the paper. $\mathrm{CH}$ was the chief investigator for the overall study, designed the study and input to the interpretation and analysis, and drafted the paper.

Funding The research was funded by the National Institute for Health Research, Health Services and Delivery Research Programme, Project reference 09/1004/04.

Competing interests All authors have completed the International Committee of Medical Journal Editors (ICMJE) uniform disclosure form at http://www.icmje.org/ coi_disclosure.pdf. JG was employed within the Primary Care Trust commissioning the dental services during the conduct of the study; $\mathrm{CH}$ and SP were on NIHR research boards during the conduct of the study. There were no other financial relationships with any organisations that might have an interest in the submitted work in the previous three years and no other relationships or activities that could appear to have influenced the submitted work.

Patient consent for publication Not required.

Ethics approval Ethics approval was obtained from the National Research Ethics Service Committee (London - Bromley, 12/L0/0205) on 5 April 2012.

Provenance and peer review Not commissioned; externally peer reviewed.

Data availability statement Data are available on reasonable request.

Open access This is an open access article distributed in accordance with the Creative Commons Attribution Non Commercial (CC BY-NC 4.0) license, which permits others to distribute, remix, adapt, build upon this work non-commercially, and license their derivative works on different terms, provided the original work is properly cited, appropriate credit is given, any changes made indicated, and the use is non-commercial. See: http://creativecommons.org/licenses/by-nc/4.0/.

\section{REFERENCES}

1 Department of Health. Nhs dental services in England. an independent review led by Professor Jimmy Steele. London: Department of Health, 2009.

2 Department of Health. Dental contract reform: prototypes. overview document. London: Department of Health, 2015.

3 Department of Health. Nhs dental contract: proposals for pilots. London: Department of Health, 2010.

4 Department of Health. NHS dental contract pilots - Learning after the first two years of piloting. London: Department of Health, 2014.

5 Department of Health. NHS dental contract pilots - Early findings. London: Department of Health, 2012.

6 Christianson J, Leatherman S, Sutherland K. Financial Incentives, Healthcare Providers and Quality Improvements. In: A review of the evidence. London: The Health Foundation, 2007.

7 O'Donnell CA, Ring A, McLean G, et al. The new GMS contract in primary care: the impact of governance and incentives on care. final report. NIHR service delivery and organisation programme 2011.

8 Langdown C, Peckham S. The use of financial incentives to help improve health outcomes: is the quality and outcomes framework fit for purpose? A systematic review. Public Health 2014;36:251-8.

9 Maisey S, Steel N, Marsh R, et al. Effects of payment for performance in primary care: qualitative interview study. $J$ Health Serv Res Policy 2008;13:133-9. 
10 Tickle M, McDonald R, Franklin J, et al. Paying for the wrong kind of performance? financial incentives and behaviour changes in national health service dentistry 1992-2009. Community Dent Oral Epidemiol 2011;39.

11 Chalkley M. Incentives for dentists in public service: evidence from a natural experiment. J Public Adm Res Theory 2010;20:i207-23.

12 Brocklehurst P, Price J, Glenny AM, et al. The effect of different methods of remuneration on the behaviour of primary care dentists. Cochrane Database Syst Rev 2013;6.

13 Hally JP, Pitts NB. Developing the first dental care pathway: the oral health assessment. Prim Dent Care 2005;12:117-21.

14 Department of Health. Dental contract reform: evaluation of the first year of prototyping 2016-2017. Department of Health: London, 2018.

15 Galloway J, Gorham J, Lambert M. The professionals complementary to dentistry: a systematic review and synthesis. London: University College London, 2002.

16 Dyer T, Robinson PG. General Health Promotion in General Dental Practice - The Involvement of the Dental Team. Part 2 - A Qualitative and Quantitative Investigation of the Views of Practice Principals in South Yorkshire. Br Dent J 2006;201:45-51.

17 Ross MK, Ibbetson RJ, Turner S. The acceptability of dually-qualified dental hygienist-therapists to general dental practitioners in southeast Scotland. Br Dent J 2007;202:146-7.

18 Nilchian F, Rodd HD, Robinson PG. Influences on dentists' decisions to refer paediatric patients to dental hygienists and therapists for fissure sealants: a qualitative approach. Br Dent $J$ 2009;207.

19 Department of Health. Delivering better Oral Health - An evidencebased toolkit for prevention. London: Department of Health, 2017.

20 Hulme C, Robinson PG, Saloniki EC, et al. Shaping dental contract reform - a clinical and cost effectiveness analysis of incentive-driven commissioning for improved oral health in primary dental care. BMJ Open 2016;2016:e013549

21 Hulme C, Robinson P, et al. Incentive: improving the organisation and delivery of dental health care to patients - innovation in commissioning and delivery of primary dental care service delivery and organisation. NIHR HS DR 2016;4.

22 Andersen RM, Davidson PL. Ethnicity, aging, and oral health outcomes: a conceptual framework. Adv Dent Res 1997;11:203-9.

23 Andersen R. A behavioral model of families' use of health services. Chicago: Center for Health Administration Studies, University of Chicago, 1968

24 Andersen R. Revisiting the behavioral model and access to medical care: does it matter? J Health Soc Behav 1995;36:1-10.
25 Goldsmith L. A critical history of Andersen's behavioral model of health services use: a reflection of how we study access to health care. Academy of Health Services Research and Health Policy 2002;19.

26 Baker SR. Applying Andersen's behavioural model to oral health: what are the contextual factors shaping perceived oral health outcomes? Community Dent Oral Epidemiol 2009;37:485-94.

27 Ritchie J, Lewis J. Qualitative research practice: a guide for social science students and researchers. London: Sage Publications Inc, 2003.

28 Department of Health. Nhs dentistry: options for change. London: Department of Health, 2002.

29 Department of Health. Primary dental care services: implementation of local commissioning. London: Department of Health, 2005

30 NHS Commissioning Board. Securing excellence in commissioning primary care, 2012. Available: https://www.england.nhs.uk/wpcontent/uploads/2012/06/ex-comm-pc.pdf

31 Forsetlund L, Bjorndal A, Rashidian A, et al. Continuing education meetings and workshops: effects on professional practice and health care outcomes. Cochrane Database Syst Rev 2009;15.

32 Schaffer SK, Sussex J, Feng Y. Incentives to follow best practice in health care. London: Office of Health Economics, 2015.

33 Department of Health. Dental contract reform engagement exercise: detailed findings. Department of Health, 2015.

34 Brocklehurst PR, Tickel M. Is skill mix Profitable in the current NHS dental contract in England? Br Dent J 2011;210:303-8.

35 Harris R, Mosedale S, Garner J, et al. What factors influence the use of contracts in the context of NHS dental practice? A systematic review of theory and logic model. Soc Sci Med 2014;108:54-9.

36 Department of Health. Dental contract reform: prototypes overview document. London: Department of Health, 2015.

37 Health Education England. The future oral and dental workforce for England. liberating human resources to serve the population across the life-course. London: Health Education England, 2019.

38 Andersen R, Davidson P. Improving Access to Care in America: Individual and Contextual Indicators. In: Andersen R, Rice T, Kominski G, eds. Changing the US health care system: key issues in health services policy and management. San Francisco: JosseyBass, 2001.

39 Dyer TA, Owens J, Robinson PG. The acceptability of care delegation in skill-mix: the salience of trust. Health Policy 2014;117:170-8.

40 Harris RV. Operationalisation of the construct of access to dental care: a position paper and proposed conceptual definitions. Community Dent Health 2013;30:94-101. 